\title{
Functional Verification of Multi-area Automatic Generation Control Coordinated System Based on Testing Platform
}

\author{
Pan Lingling ${ }^{a}$, Li Feng ${ }^{b}$ and Liu Jiantao ${ }^{c}$ \\ China Electric Power Research Institute, Nanjing 210003, China \\ apanlingling@epri.sgcc.com.cn, ${ }^{b}$ lifeng2@ epri.sgcc.com.cn, ${ }^{C}$ liujiantao@epri.sgcc.com.cn
}

\begin{abstract}
Keywords: UHVDC, Multi-area AGC, Testing Platform, Frequency coordination control, Functional Verification.

Abstract. A function verification method of multi-area AGC coordinated system is proposed in this paper. Based on the power grid model of testing platform, the external multi-area AGC simulation system is build. A joint simulation system is formed by the simulated AGC system and the tested AGC system, which covers the whole national power grid. Data simulation layer of testing platform provides the wide-area substation communication service to collect PLC control instructions from multi-area AGC, and power grid simulation layer of testing platform provides event handling process to conduct the control instructions. The method can test multi-area AGC coordinated system, and improves the accuracy of active power response.
\end{abstract}

\section{Introduction}

As the development of UHV system in China, interaction between the supply and receiving end becomes more and more prominent, and the influences of system fault on power grid operation extend the scope[1-4]. High power Loss due to UHV DC blocking make serious impact on safe operation of the receiving system, and the system frequency can fast recover using multi-area AGC[5-8]. To ensure the accuracy of active power response, it needs to test multi-area AGC function under situation of high power loss.

Power grid simulation system of testing platform provides the whole power grid model[9], and simulates the external multi-area AGC system. It provides a complete operation environment for functional verification, including operation mode change, active power allocation, ramp speed, and governor response. And then testing platform verifies the function of multi-area AGC coordinated control, and improves the accuracy of active power response.

\section{Functional Verification Framework}

Frequency coordination control software is deployed on the East China power dispatching system to deal with high power loss caused by UHV DC blocking. AGC module is also deployed on power dispatching system, and external multi-area AGC simulation system is provide by testing platform. The multi-area AGC coordinated function is verified by setting various operation scenarios, and controlling unit response. The testing platform can be divided into four layers, including power system simulation layer, data simulation layer, dispatching automation system layer, and testing control layer. It provides operation data for AGC system.

\section{Power grid model and simulation}

When high power loss occurs in regional power grid, the frequency coordination control software can rapidly restore the frequency by multi-area AGC. The testing platform adopts the East China Power Grid model, including East China dispatching system, Jiangsu dispatching system, Zhejiang dispatching system, Fujian dispatching system, Anhui dispatching system, and Shanghai dispatching system.

Based on the East China power grid model, testing platform simulates high power loss scenario, and sends power grid operation data to dispatching system. The frequency coordination control software responds to power grid emergency operation status and achieves coordinated AGC in multiple control areas. Testing platform builds tie line and unit control model for each control area. 
The overall framework of functional verification is shown in Figure 1.

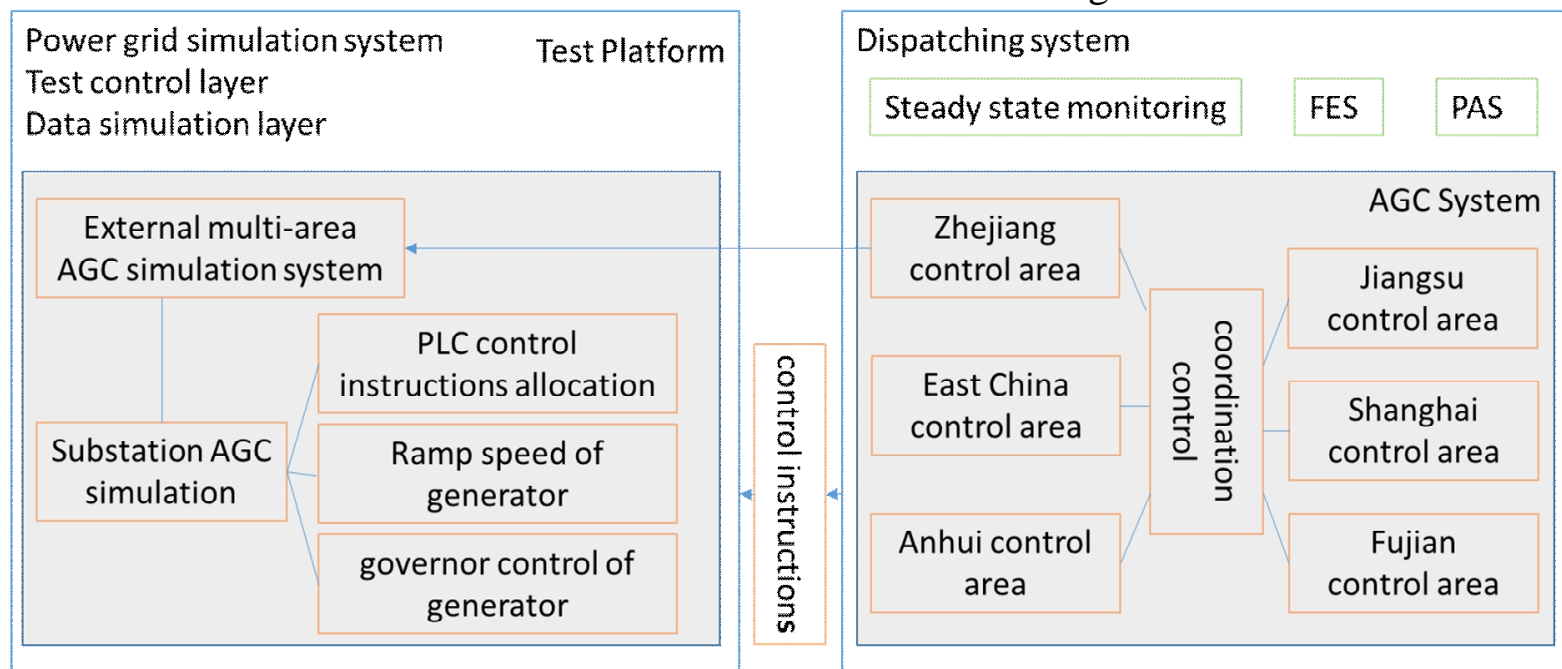

Fig. 1 Functional Verification Framework of multi-area AGC

\section{The closed-loop simulation of AGC control instructions}

Frequency coordination control software calculates the ACE exchange value of multi-area AGC system according to the power grid operation status, and controls the ACE value to lead the AGC response in different control areas. The AGC system issues PLC control instructions to the power plant, and the testing platform receives the instructions and performs closed-loop simulation to support the whole process of frequency control simulation, and then verify the coordination control effect of multi-area AGC.

The testing platform provides substation AGC simulation. The testing platform receives control instructions from AGC systems in different control areas, and then simulates allocation methods from PLC control instructions to unit control commands. Based on ramp speed and governor characteristics, testing platform simulates the active power control process. The control results are finally reflected in power flow changes, which are fed back to the dispatching system to drive the next cycle control of the frequency coordination control.

The long-term frequency control process is simulated through the closed-loop simulation of AGC control instructions. It is beneficial to evaluate the function correctness of frequency coordination control software from the control process and control results.

\section{Test Scenes}

The frequency coordination control software, as application software, needs to be able to cope with different abnormal conditions, such as abnormal response of the substation AGC, abnormal AGC response in control area, abnormal response of generator. Testing platform provides various abnormal scenes.

\section{Abnormal response of substation AGC}

The substation AGC receives AGC control instructions from the dispatching system, and allocates the PLC control instructions to generators, and then the generators respond to the control instructions. In the test process, a variety of abnormal responses of substation AGC are given, including abnormal active power distribution, and abnormal response to control instructions. Abnormal generator response can also be obtained by setting generator parameters, including changing the ramp rate of multiple or single generator, non-response control instructions, reverse response control instructions, and abnormal response value.

\section{Abnormal AGC response in control area}

Testing platform can build a specified control area model in the power grid simulation system to provide AGC simulation, and set an expected deviation on active control of the control area. Abnormal AGC response cases are added during the control process, including unsuccessful ACE delivery from frequency coordination control software, AGC system fault, and slow AGC response. 


\section{Abnormal fluctuation of regional load}

When high power loss and abnormal fluctuation of regional load occur together, grid instability will be aggravated and the frequency coordination control software needs to be able to cope with this scenario. Testing platform provides customized control model of regional load, which can customize multiple regional loads, and give load curves in each area. It simulates abnormal fluctuations of regional load to test the frequency coordination control function.

\section{Test Example and Results}

Functional verification of frequency coordination control software can be performed in several aspects, including ACE differential computation (ADI), calculation method, control mode for standby emergency power support, measurement data processing method, operation condition determination methods, interaction with scheduling data, active power support periodicity, and AGC adaptive control.

A test example is given in this paper. When a DC blocking fault occurs, the system loss 1600 MW of active power, and the system frequency drops to $49.830 \mathrm{~Hz}$. Dynamic ACE allocates 1000 MW active power shortage to each control area to support the frequency recovery. When multi-area AGCs run, the system frequency curve is shown as Figure 2. The frequency drops to $49.82 \mathrm{~Hz}$, and then returns to $49.98 \mathrm{~Hz}$ after 5 minutes and keeps in this state. The ACE curves for each control area is shown in Figure 3.

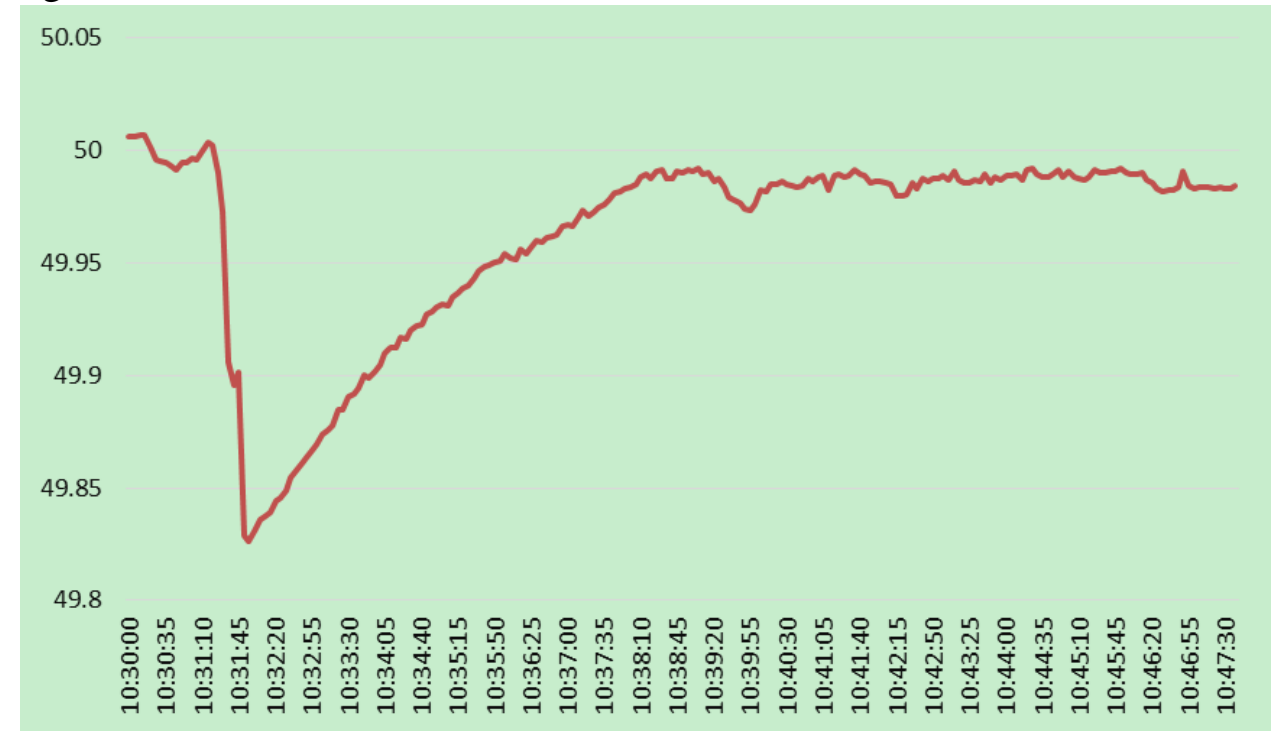

Fig. 2 Frequency curve of power system

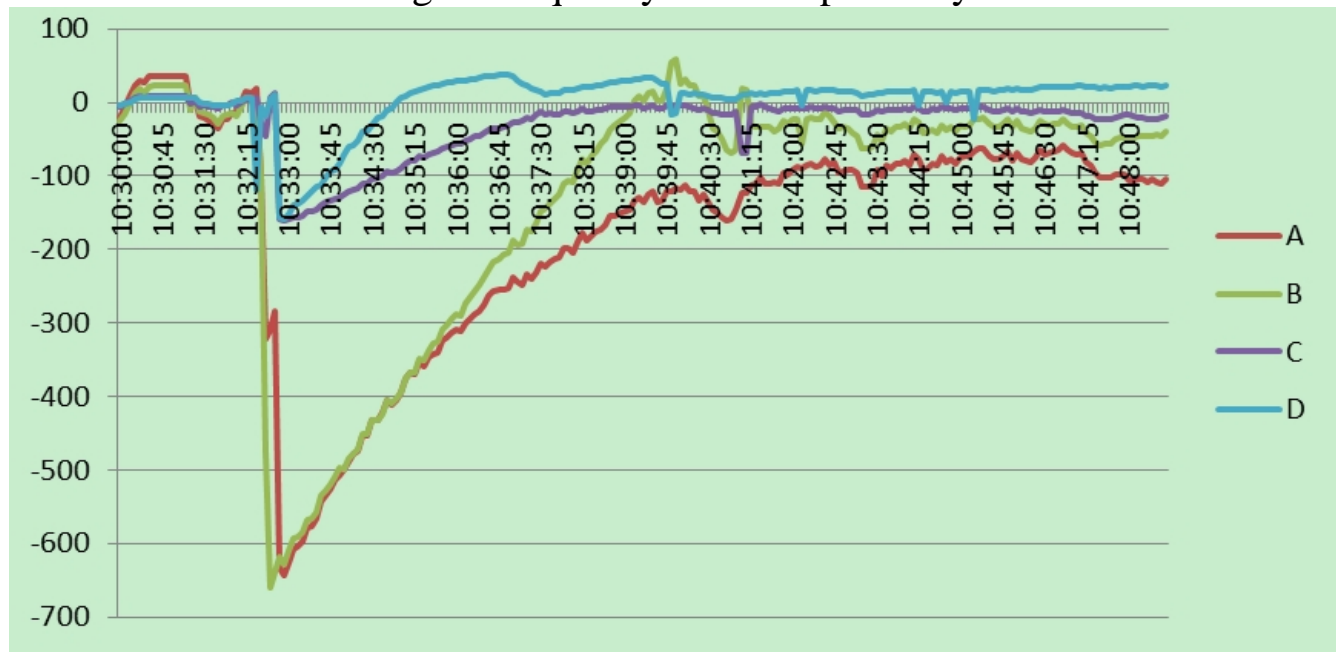

Fig. 3 ACE curves for each control area 


\section{Conclusions}

Testing platform provides a closed-loop control simulation environment for multi-area AGC functional test under the condition of high power loss, and provides various abnormal conditions to verify the accuracy of active power response.

\section{Acknowledgements}

This work was financially supported by National Key R\&D Program of China (2017YFB0902605) and Science and Technology Project "Key Technologies and application of Dispatching Control System Testing and Integrated Operation and Maintenance" of SGCC.

\section{References}

[1] LI Weidong, JIN Cuicui, WEN Kerui, et al. Preliminary research on active frequency response control under high-power loss[J]. Automation of Electric Power Systems, 2018,42:1-9.

[2] LI Zhaowei, WU Xuelian, ZHUANG Kanqin, et al. Analysis and reflection on frequency characteristics of East China grid after bipolar locking of" $9 \cdot 19^{\prime \prime}$ Jinping-Sunan DC transmission line[J]. Automation of Electric Power Systems,2017,41(7):149-155.

[3] LI Weidong, CHANG Yekui, CHEN Zhaoqing, et al. Dynamic contents of area control error[J]. Automation of Electric Power Systems, 2016, 40(24):146-150 .

[4] MU J, WU W, SUN H, et al. An online intelligent alarm processing system based on abductive reasoning network[C]. IEEE Power and Energy Society General Meeting, July 22-26, 2012, San Diego, CA, USA:5p.

[5] UKIL A, ZIVANOVIC R. Application of abrupt change detection in power systems disturbance analysis and relay performance monitoring[J]. IEEE Trans on Power Delivery, 2007, 22(1):59-66.

[6] TENG Xialiang, GAO Zonghe,ZHU Bin, et al. Requirements Analysis and Key Technologies for Automatic Generation Control for Smart Grid Dispatching and Control Systems[J] . Automation of Electric Power Systems, 2015, 39(1):81-87.

[7] ZHAO Chuan, ZHU Tao, YE Hua. Research and realization of coordinated automatic generation control based on multi-control zone[J]. Power System Protection and Control, 2014, (16):47-51.

[8] CHENG Limin, LI Xingyuan. Load frequency control in multi-area AC/DC interconnected power system[J].Power System Protection and Control, 2011,39(7):56-62.

[9] LI Feng, ZHUANG Wei-jin, WANG Yong, et al. PSS/E-based simulation system design for functional verification on the power dispatching automation system[J]. Electric Power, 2014, 47(1):66-71. 